\title{
Discharge Medication Reconciliation for Patients Being Discharged to a First Nations Reserve*
}

\author{
Jaris Swidrovich
}

\section{INTRODUCTION}

$\mathrm{T}$ The Canadian Patient Safety Institute defines medication reconciliation as "a formal process in which healthcare providers work together with patients, families, and care providers to ensure that accurate, comprehensive medication information is communicated consistently across transitions of care". ${ }^{1}$ Following the medication reconciliation process is known to result in fewer adverse drug events and, therefore, positive health outcomes for patients. ${ }^{1}$ In 2010, Accreditation Canada, a nonprofit organization dedicated to health care improvement, mandated Canadian hospitals to have an established medication reconciliation program in at least one clinical area in order to become accredited. ${ }^{2}$ Certainly, Canadian hospitals face a number of challenges in meeting this accreditation standard, including the need for adequate staff and resource allocation. However, geographic, jurisdictional, and cultural differences contribute added complexity for patients coming from and being discharged to First Nations reserves, because registered First Nations persons and recognized Inuit are medically insured by the federal government, rather than by the provincial governments, which cover all nonregistered First Nations and nonInuit persons. ${ }^{3}$ Caught between the federal and provincial health systems and accessing care in both municipalities (under provincial jurisdiction) and reserves (under federal jurisdiction), First Nations and Inuit individuals are subject to drastic service and funding inequities, which have direct impacts on all of the social determinants of health and health care services. ${ }^{4}$ The Calls to Action of the Truth and Reconciliation Commission of Canada stress the importance of closing the gaps in health outcomes between Indigenous (First Nations, Métis, and Inuit) and non-Indigenous

*This article intentionally uses gender-inclusive language (e.g., "themselves" instead of "him/herself") to be inclusive of nonbinary and Two Spirit individuals. people in Canada. 5 Pharmacists, the most accessible of health care professionals and often ranked as the most trusted, are uniquely and ideally situated in Canada's health care system to both respond to and proactively prevent inequities in the health outcomes experienced by Indigenous people in Canada. ${ }^{6,7}$ In particular, discharge medication reconciliation performed by hospital pharmacists is likely to contribute to positive health outcomes for all hospitalized patients, including First Nations individuals being discharged to a reserve. ${ }^{8}$

About $50 \%$ of all First Nations people in Canada live on reserve. ${ }^{9}$ Few pharmacies exist on reserves across Canada, leaving the people of most reserves with no option but to use the services of a pharmacy in a nearby city, town, or community. In addition, some reserves have contracts with particular pharmacies that will dispense and ship medications to the reserve at a certain frequency. As such, it is possible, and indeed common, for many First Nations people living on reserve to never interact with a pharmacist face-to-face. Despite the criticality of relationships in Indigenous communities and in health service delivery to First Nations people, it may be difficult for pharmacies and pharmacists to provide relationship-based care to First Nations individuals living on reserves without a pharmacy, especially in circumstances where the First Nations patient and the pharmacist have limited interactions. Conversely, in an acute care environment, there is significant opportunity to ensure that First Nations individuals coming from and/or being discharged to a reserve receive care from a pharmacist.

The objective of this article is to bridge a gap in the literature by highlighting unique challenges experienced when providing medication reconciliation services for patients coming from and/or being discharged to a First Nations reserve and to offer practical suggestions for hospital pharmacists to use in their care of such individuals (summarized in the form of a checklist in Box 1). 


\section{Box 1. Discharge Medication Reconciliation Checklist for Patients Being Discharged to a Reserve Complete medication assessment}

Have all medications and medicines been assessed?

Patient may not be eligible for or may not have access to provincial comprehensive medication assessment program by community pharmacist. Is or will the patient be taking any traditional First Nations medicines?

Is there any published information about the traditional medicine(s)?

Are any precautions required with co-administration of traditional medicines and the patient's other medications?

Has enough supply been prescribed to ensure no gaps in therapy?

Have the patient's family and/or friends been included in the assessment and education (with the patient's permission)?

\section{Community pharmacy}

Does the reserve have a contract with a particular community pharmacy?

What is the delivery schedule of the pharmacy that services the reserve?

Can/should the hospital send an interim supply of medications home with the patient?

Does the pharmacy have the required stock to send an interim supply for the patient?

\section{Medication insurance coverage}

Does the patient have a First Nations status card (and therefore an NIHB billing number)?

Does the patient have other medication coverage (e.g., work insurance plan, through spouse or parent, social assistance)?

Will any medication(s) require prior approval through NIHB? If yes, check NIHB formulary to ensure the medication(s) are listed.

\section{Provision of physician services on the reserve}

What is the schedule for physician services to the reserve?

Does the discharge medication quantity and/or number of refills need to be increased?

\section{Language services}

Are language services required to ensure that the patient understands the discharge medication plan?

Are there any written materials about the medications and/or disease state available in the patient's primary language?

\section{Interprofessional and community collaboration}

Do any other professionals or community members need to be consulted to ensure seamless care?

$\mathrm{NIHB}=$ Non-Insured Health Benefits.

\section{PRACTICE DESCRIPTION AND IMPLICATIONS}

\section{Complete Medication Assessment}

Hospital pharmacists not only have the opportunity and ability to establish a relationship with First Nations patients coming from and/or being discharged to a reserve but can also provide such patients with comprehensive and quality care. Instead of the typical concentrated focus on medications that have been newly started, stopped, and/or adjusted during the hospitalization, medication reconciliation for a First Nations person coming from and/or being discharged to a reserve should involve critical attention to all medications that the person is taking. Often, status First Nations individuals are ineligible for provincially funded medication review programs (e.g., Saskatchewan Medication Assessment Program) because health care for First Nations people with a status card (and for "recognized Inuit") is federally funded. As such, it is unrealistic to expect that a status First Nations person will receive a complete and comprehensive medication assessment from a community pharmacist in the absence of a federal reimbursement structure through the Non-Insured Health Benefits (NIHB) program.

When participating in the best possible discharge medication reconciliation process for a First Nations individual being discharged to a reserve, pharmacists should first check whether a best possible medication history (BPMH) was completed on admission to hospital. The BPMH is a systematic process of interviewing the patient/family and reviewing at least one other reliable source of information to obtain and verify information about all medications (prescribed and nonprescribed) that a patient is using. ${ }^{1}$ Completion of a BPMH is critical, as the information obtained may differ from what appears in the patient's records. ${ }^{1}$ It is important to note that prescriptions filled by a First Nations person that were billed to the federal NIHB program may not appear on provincial medication record databases if the individual did not also, at the time of dispensing, present a provincial health card along with their prescription and status card. As such, due diligence must be exercised when conducting a BPMH for First Nations patients, particularly as it relates to prescription medications that may not appear in provincial databases.

Although it is not unusual for family members, friends, and/or other visitors to be included in a hospital pharmacist's interactions with any hospitalized patient, the intentional inclusion of such individuals in interactions with First Nations patients coming from and/or being discharged to a reserve is encouraged-with the patient's permission, of course. Wahkotowin (the Cree word for "kinship") is a common 
characteristic of First Nations' world view and family life. Additionally, the concept of health and wellness is not limited to the individual alone but also includes others who are close to the individual. In the setting of medication reconciliation processes, more complete medication and health information is likely to be obtained with the inclusion of individuals who are close to the First Nations patient in terms of relationships and connectedness. As such, engagement with the people, family, and community around and close to a First Nations patient is considered as important in the discharge medication reconciliation process as engagement with the patient themself.

Although population-wide generalizations cannot be made, there is often a greater likelihood that on-reserve First Nations individuals (relative to First Nations persons growing up and/or living off-reserve) will use, or at least have a strong appreciation for, traditional medicines and traditional approaches to health and wellness. Therefore, the use of traditional medicines and/or practices should be considered in the discharge medication reconciliation processes for an individual being discharged to a reserve. Traditional First Nations medicines and the practices related to understanding and disseminating knowledge about their use are not often (if ever) indexed and recorded in the ways that Western medications and practices are documented. As such, trying to retrieve information about traditional First Nations medicines and practices from the common and typical sources familiar to pharmacists may yield minimal or no results. Furthermore, few traditional First Nations medicines are indexed in alternative resources, such as the Natural Medicines Comprehensive Database (https://naturalmedicines.therapeuticresearch.com/), so pharmacists should be prepared to conduct discharge medication reconciliation without the information and literature about such medicines that would typically be available for mainstream prescription and nonprescription medications. ${ }^{10}$

\section{Community Pharmacy}

If the patient does not already have a regular pharmacy (either one where they can pick up their own medications or one that will deliver medications to the reserve), the hospital pharmacist is encouraged to determine whether the reserve to which the patient is being discharged has a contract with a particular community pharmacy. If, after conversation with the patient, the pharmacist has been unable to determine the contract status of the patient's reserve, it may be necessary to make a phone call to either the reserve's health centre or Band Office, the most appropriate contacts for all reserves. Upon determining which pharmacy will dispense the patient's medications, the hospital pharmacist should contact that pharmacy for the fax number to which discharge medication reconciliation information and the prescription(s) may be sent. Additionally, it is suggested that the hospital pharmacist inquire whether the pharmacy has the necessary stock for the patient's prescriptions and confirm the dates and times when the pharmacy makes deliveries to the reserve, to ensure that the patient will receive all needed medications on time, without any gaps in therapy. Occasionally, it may be most convenient and economical to send the patient home to their reserve with some of the hospital's stock of medication, rather than keeping the patient in hospital while their contracted community pharmacy acquires, dispenses, and delivers the medications.

\section{Medication Insurance Coverage}

When confirming insurance coverage for medications, the contracted pharmacy will, of course, need the patient's NIHB number and information regarding any other medication coverage (e.g., through work, parent, or spouse). If the NIHB program will be the payer, or partial payer, of the person's prescriptions, the hospital pharmacist should first review the NIHB formulary online, because this formulary is not always consistent with a particular province's formulary. Furthermore, "limited use" medications on the NIHB formulary may not be consistent with provincial practices regarding which medications require special/prior approval. An NIHB requirement for prior approval for any of the patient's medications may cause a significant delay in either the discharge date for the patient or the date when the medications are dispensed to the patient at no cost. Once again, it may be most convenient and economical, for both the patient and the hospital, to discharge the patient home to reserve with enough supply from the hospital's stock to ensure there are no gaps in medication therapy while the prior approval process is adjudicated, which may take a few days.

\section{Provision of Physician Services to the Reserve}

The timing and frequency of physician services to reserve communities must also be considered during the discharge process. Physician services are rarely offered 7 days a week, and in many cases occur as infrequently as 1 day or 1 half-day every 1 to 2 weeks. For patients whose future care will require a physician's monitoring and/or intervention, the hospital pharmacist should collaborate with hospital and/or community prescribers to ensure the patient is discharged with a sufficient quantity of medication and any necessary refills to avoid potential gaps in pharmacotherapy that could arise because of infrequent provision of physician services to the reserve community. If the patient does not know the frequency of physician services to the community, a phone call to the reserve's health centre or Band Office may be warranted.

\section{Language Services}

Beyond both the expected and less familiar logistical tasks to consider for First Nations individuals being discharged from hospital to a reserve, ethnocultural differences should also be considered. Pharmacists should seek or be aware of ethnoculturally appropriate services, information, and supports that may be of benefit for First Nations patients. For example, it may be necessary 
to arrange language services for the patient and/or family, not only during the hospital stay but also for all discharge processes and discharge teaching. Given that many First Nations individuals living on reserve may have minimal to no access to a pharmacist, and given that access to a pharmacist who speaks the individual's First Nations language may be even more limited, discharge medication reconciliation and medication teaching activities should include interpretation services as needed. Collaboration with speakers of the First Nations languages commonly spoken by the patients and communities often seen within a particular hospital is also encouraged, to help in the development of written and/or verbal medication information in the particular languages, especially for high-risk medications (e.g., warfarin).

\section{Interprofessional and Community Collaboration}

In the spirit of interdisciplinarity, pharmacists may collaborate with other health professionals when performing discharge medication reconciliation processes for individuals being discharged to a reserve. If the hospital has its own First Nations health service, the pharmacist is encouraged to request the service to participate in the admission and discharge of all First Nations individuals. Such specialized health services often have Elders on staff, as well as health navigators, language keepers, social workers, and others with particular expertise. For hospitals with no such service, the pharmacist and other members of the health care team are encouraged to consider which culturally relevant services might be available for the patient after discharge to the reserve, if the patient so desires.

If the discharged patient will need daily transportation to and from a community pharmacy (e.g., for daily witnessed dosing of methadone), consultation with a health navigator or social worker may be required to make the necessary arrangements. Notably, pharmacists should be aware of the stigma associated with the term "social worker" in some (though not all) First Nations communities. More specifically, for some First Nations people and communities, the term "social worker" is associated with traumatic situations and events, such as the residential school system and the Sixties Scoop, when social workers apprehended children, often without the permission of the family or First Nations authorities. Only through engagement with the patient, family, community, health care team, and local social workers themselves will pharmacists be able to ascertain whether there is any concern with using this term.

\section{CONCLUSION}

Despite being experts in medication reconciliation on admission to, transfer between, and discharge from hospitals, pharmacists should be cognizant of the unique experiences of First Nations patients coming from and/or being discharged to a reserve. Positive interactions with health care providers such as pharmacists and a better understanding of their role in improving patient outcomes can encourage First Nations individuals to consider becoming health care providers themselves. First Nations patients will continue to be admitted from and discharged to reserves, so a focus on the unique features described in this article is likely to improve the patient outcomes of and reduce disparities for First Nations patients.

\section{References}

1. Medication reconciliation. Edmonton (AB): Canadian Patient Safety Institute; 2016 [cited 2019 May 5]. Available from: https://www. patientsafetyinstitute.ca/en/Topic/Pages/medication-reconciliation(med-rec).aspx

2. Accreditation Canada; Canadian Institute for Health Information; Canadian Patient Safety Institute; Institute for Safe Medication Practices Canada. Medication reconciliation in Canada: raising the bar. Progress to date and the course ahead. Ottawa (ON): Accreditation Canada; 2012 [cited 2019 Feb 20]. Available from: https://www.ismp-canada.org/ download/MedRec/20121101MedRecCanadaENG.pdf

3. Non-Insured Health Benefits (NIHB) Program - general questions and answers. Ottawa (ON): Government of Canada; 2018 [cited 2019 May 3]. Available from: https://www.canada.ca/en/indigenous-services-canada/ services/first-nations-inuit-health/non-insured-health-benefits/benefitsinformation/non-insured-health-benefits-nihb-program-generalinformation-questions-answers-first-nations-inuit-health-canada.html

4. I am a witness - background. Ottawa (ON): First Nations Child \& Family Caring Society; [cited 2019 May 5]. Available from: https:// fncaringsociety.com/i-am-witness-background

5. Honouring the truth, reconciling for the future: summary of the final report of the Truth and Reconciliation Commission of Canada. Winnipeg (MB): Truth and Reconciliation Commission of Canada; 2015 [cited 2019 Feb 20]. Available from: http://www.trc.ca/assets/pdf/Honouring the_Truth_Reconciling_for_the_Future_July_23_2015.pdf

6. Berardi C. PAM 2016: changing roles help pharmacists do more for patients. Ottawa (ON): Canadian Pharmacists Association; 2016 [cited 2019 Feb 20]. Available from: https://www.pharmacists.ca/news-events/ cpha-blog/pharmacist-awareness-month-2016/

7. Marotta R. Pharmacists remain among most trusted professions. Pharm Times. 2016 Dec 21 [cited 2019 Feb 20]. Available from: https:// www.pharmacytimes.com/news/pharmacists-remain-among-mosttrusted-professions

8. Medication reconciliation: statement on the role of the pharmacist (2009). Ottawa (ON): Canadian Society of Hospital Pharmacists; 2009 [cited 2019 May 5]. Available from: https://www.cshp.ca/sites/default/files/files/ Advocacy/Med\%20Incidents/Statement\%20-\%20Med\%20Rec\% 202009.pdf

9. Aboriginal peoples in Canada: First Nations people, Métis and Inuit. Ottawa (ON): Statistics Canada; 2018 [cited 2019 Feb 20]. Available from: https://www12.statcan.gc.ca/nhs-enm/2011/as-sa/99-011-x/99-011x2011001-eng.cfm

10. Hsu PP. Natural medicines comprehensive database [review]. J Med Libr Assoc. 2002;90(1):114.

Jaris Swidrovich, BSP, PharmD, is with the College of Pharmacy and Nutrition, University of Saskatchewan, Saskatoon, Saskatchewan.

Competing interests: None declared.

Address correspondence to:

Dr Jaris Swidrovich

College of Pharmacy and Nutrition

University of Saskatchewan

Health Sciences Building (E-Wing), Room 3124

104 Clinic Place

Saskatoon SK S7N $2 Z 4$

e-mail: jaris.swidrovich@usask.ca

Funding: None received. 